\title{
Ventilator-associated events: prevalence and mortality in Japan
}

\author{
Susumu Nakahashi ${ }^{1}$, Hiroshi Imai ${ }^{1}$, Hideaki Imanaka ${ }^{2}$, Shinichiro Ohshimo ${ }^{3}$, Tomoko Satou ${ }^{4}$, \\ Masanori Shima $^{5}$, Masami Yanagisawa ${ }^{6}$, Chizuru Yamashita ${ }^{7}$, Toru Ogura $^{8}$, Tomomi Yamada $^{9}$, \\ Nobuaki Shime ${ }^{3}$; for the VAEs Review Committee in the Japanese Society of Intensive Care Medicine \\ (JSICM)
}

${ }^{1}$ Department of Emergency \& Critical Care Medicine, Mie University Graduate School of Medicine, Mie, Japan; ${ }^{2}$ Emergency and Critical Care Medicine, Tokushima University Hospital, Tokushima, Japan; ${ }^{3}$ Department of Emergency and Critical Care Medicine, Graduate School of Biomedical \& Health Sciences, Hiroshima University, Hiroshima, Japan; ${ }^{4}$ Intensive Care Unit, Akita University Hospital, Akita, Japan; ${ }^{5}$ Department of Nursing, Mitsubishi Kyoto Hospital, Kyoto, Japan; ${ }^{6}$ Department of Medical Engineering, National Hospital Organization Kyoto Medical Center, Kyoto, Japan; ${ }^{7}$ Department of Anesthesiology and Critical Care Medicine, Fujita Health University School of Medicine, Aichi, Japan; ${ }^{8}$ Clinical Research Support Center, Mie University Hospital, Mie, Japan; ${ }^{9}$ Department of Medical Innovation, Osaka University Hospital, Osaka, Japan Contributions: (I) Conception and design: H Imai, N Shime; (II) Administrative support: S Nakahashi, H Imai, N Shime; (III) Provision of study materials or patients: S Nakahashi, H Imanaka, S Ohshimo, T Satou, M Shima, M Yanagisawa, C Yamashita; (IV) Collection and assembly of data: S Nakahashi, H Imanaka, S Ohshimo, T Satou, M Shima, M Yanagisawa, C Yamashita, H Imai, N Shime; (V) Data analysis and interpretation: S Nakahashi, T Ogura, T Yamada, H Imai, N Shime; (VI) Manuscript writing: All authors; (VII) Final approval of manuscript: All authors.

Correspondence to: Susumu Nakahashi. Department of Emergency \& Critical Care Medicine, Mie University Graduate School of Medicine, 2-174, Edobashi-Tsu, Mie 5148507, Japan. Email: n14w4@s3.dion.ne.jp; Nobuaki Shime. Department of Emergency and Critical Care Medicine, Graduate School of Biomedical \& Health Sciences, Hiroshima University, 1-2-3, Kasumi, Minami-ku, Hiroshima 7348551, Japan.

Email: nshime@hiroshima-u.ac.jp.

Background: In 2013, the Centers for Disease Control and Prevention (CDC) issued the concept of the ventilator-associated events (VAEs) as a quality indicator (QI) in the intensive care unit (ICU). A number of studies have been conducted in the United States and other Western countries to evaluate its practicality. However, information on VAEs in non-Western countries is scarce. The purpose of this preliminary study was to illuminate the incidence and associated mortality rate of VAEs in Japan, as a first step in the effort to determine its practicality.

Methods: We conducted a multi-center, retrospective review of patient medical record using VAEs surveillance algorithm. We analyzed 785 patients with $\geq 2$ days of mechanical ventilator (MV), admitted to the ICU at seven urban hospital in Japan. The prevalence of VAEs, including its three subtypes, and in-ICU mortality were researched.

Results: Forty-nine VAEs were identified, affecting 5.7\% of patients requiring MV for $\geq 2$ days and 6.4 per 1,000 MV days. Mortality in those who developed VAEs was 42.9\%, significantly higher than the rest of the cohort (vs. 15.4\%, $\mathrm{P}<0.001$ ). The overall equivalent distribution of the three VAEs subtype incidences was evaluated: the incidences of VAC only, IVAC only and PVAP were 2.20, 1.90 and 2.29 per 1,000 MV days respectively $(\mathrm{P}=0.933)$. However, VAE etiology and mortality was facility dependent. ICUs with a large proportion of surgical patients and more severe cases tended to have increased VAE incidence, with a converse decrease in closed ICUs.

Conclusions: The prevalence of VAEs appears low in Japanese ICUs. Nonetheless, mortality was substantially higher in patients who developed VAEs. Although some potential indices of VAEs are suggested to serve as QIs, additional studies to elaborate its practicality would further be required.

Keywords: Ventilator-associated events (VAEs); surveillance; quality indicator (QI); patient safety; intensive care unit (ICU)

Submitted Aug 11, 2017. Accepted for publication Nov 06, 2018.

doi: $10.21037 /$ jtd.2018.12.40

View this article at: http://dx.doi.org/10.21037/jtd.2018.12.40 


\section{Introduction}

In 2013, the Centers for Disease Control and Prevention (CDC)/the National Healthcare Safety Network (NHSN) issued new surveillance algorithms for ventilator-associated events (VAEs) (1). VAEs are comprehensive respiratory complications that occur in conjunction with use of mechanical ventilation (MV). The VAE definition was developed as a new indicator of quality of care in intensive care unit (ICU) to replace previously used ventilatorassociated pneumonia (VAP) (2-4). Its advantages are all objective, simple, and potentially automatable. CDC/ NHSN has placed emphasis on shifting surveillance away from traditional VAP to VAEs to eliminate subjectivity in surveillance (5-8). Because of promulgation of VAEs, a number of studies have been conducted in the United States and other Western countries (9-14) to evaluate its practicality as a quality indicator (QI) in ICU. The recent reports demonstrated that VAEs were associated with prolonged use of MV $(11,15,16)$ prolonged ICU stay $(2,12,17)$ and increased mortality $(15,18,19)$.

However, information on VAEs in non-Western countries is scarce, with there being no literature on them, except for a few reports (19-21). The value of VAEs as QIs is uncertain. The extent to which VAEs affect patients is unknown. Evidence supporting its use by clinicians in nonWestern countries is limited. VAEs should also be evaluated for its practicality as QIs and potential impact on future quality improvement outside Western countries. The first step in this effort is to clearly determine the incidence and mortality rate of VAEs.

The purpose of this preliminary study was to investigate the incidence and its associated factors, and mortality rate of VAEs in Japan.

\section{Methods}

The study was reviewed and approved by the Institutional Review Board (IRB) of the Mie University Graduate School of Medicine. The IRB approved an opt-out and waiver of consent for use in this project due to the low risk of injury, subject burden, and disclosure of personal information (IRB number: 1468). To research the prevalence of VAEs in Japanese ICUs we conducted a multi-center, retrospective review of patient medical records. We analyzed the medical records of patients who presented to seven affiliated ICUs (consisting of five universities and two community hospitals) between January 2014 and December 2014.

\section{Definitions and measures}

VAEs and ventilator-associated conditions (VACs) were defined as per CDC guidelines $(1,5)$ : the baseline period was defined as the two calendar days immediately preceding the first day of increased daily minimum (positive endexpiratory pressure) PEEP or (fraction of inspired oxygen) $\mathrm{FiO}_{2}$. A period of ventilator stability or improvement was defined as $\geq 2$ calendar days of stable or decreased daily minimum $\mathrm{FiO}_{2}$ or PEEP values. After a period of ventilator stability or improvement, a VAE was noted if the patient had at least one of the following indicators of worsening oxygenation: (I) an increase in the daily minimum $\mathrm{FiO}_{2}$ of $\geq 0.2$ over the daily minimum $\mathrm{FiO}_{2}$ in the baseline period sustained for $\geq 2$ calendar days and/or (II) an increase in the daily minimum PEEP values of $\geq 3 \mathrm{cmH}_{2} \mathrm{O}$ over the daily minimum PEEP in the baseline period sustained for $\geq 2$ calendar days. Infection-related ventilator-associated complication (IVAC) was defined as the presence of VAC in a patient who also fulfilled the following criteria: $\geq 4$ days of requiring one or more new antibiotics and any one of the following within 2 days before or after the day of VAC onset (excluding the first 2 days of $\mathrm{MV}$ ): white blood cell count of $\leq 4,000$ or $\geq 12,000$ cells $/ \mathrm{mm}^{3}$, temperature of $<36$ or $>38^{\circ} \mathrm{C}$. Possible and probable ventilator-associated pneumonia (PVAP) was defined as the presence of IVAC in a patient who also had evidence of pneumonia (e.g., positive culture of lower respiratory secretions, positive diagnostic test for selected respiratory viruses, and objectively defined purulent lower respiratory secretions).

Regarding the subtypes of VAEs, VAC only (same meaning as non-infectious complication) was defined as a patient meeting the VAC criteria not IVAC. Similarly, IVAC only (same meaning as infectious complications other than pneumonia) was defined as a patient meeting the IVAC criteria but not PVAP (15).

The incidence rate and distribution of VAEs and each subtype of VAEs were determined. Then, we investigated if VAEs were related to mortality. We identified the subtypes of VAEs associated with high mortality. Finally, we explored what type of facilities can have high incidence of VAE.

\section{Statistics}

Of the 4,541 patients admitted to the ICU during the study period, 1,464 patients ( $\geq 18$ years of age) were mechanically ventilated. Of these, 785 patients received $M V$ for $\geq 2$ days were analyzed in this study (Figure 1). Continuous variables 


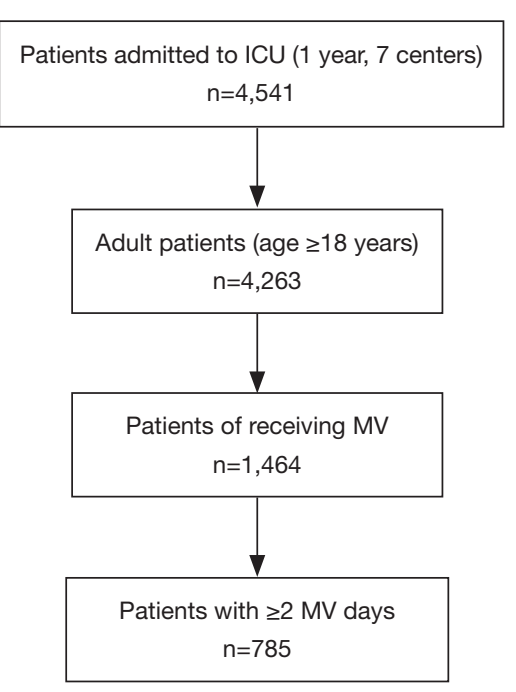

Figure 1 Schematic of the patient selection process.

were summarized using mean and standard deviation (SD). Categorical variables were numerically summarized and percentages presented where appropriate. The VAEs and No VAE groups were compared by the Student $t$-test for continuous variables and by chi-square test for categorical variables. One way ANOVA and chi-square test were used to compare the prevalence and mortality among VAE subtypes. The VAE incidence and mortality rate among the facilities were compared using chi-square test and multiple testing controlled by the Benjamini-Hochberg procedure (22). To determine the type(s) of facility with high VAE incidence, VAE prevalence was analyzed by the Generalized linear model (GLM) to test the impact of age distribution, overall ICU severity (APACHE II score), and unit type. The rationale for these thresholds of stratification are summarized as follows: (I) 65 years of age as the threshold: First, based on survey results of ICU functions in Japan, the average age of ICU patients was 66 years old (23). Second, pre-elderly is defined as 65 years old in Japan. Third, the median value of this study was 65 years. (II) APACHE II score of 20 as the threshold: An APACHE II score of 20 is generally observed as a dividing ridge as the predicted mortality rate is $20 \%$ and $40 \%$, respectively, for a score of 20 or less and 20 or more, which is a large difference (24). (III) Surgical patient proportion: In a Japanese standard ICU, the percentage of surgical patient is about 45 (23). (IV) ICU type: previous studies have shown that the prognosis of ICU patients is better in closed ICU than in nonclosed (open/semi-open) ICU (25). All patients in a closedICU are cared for by intensivist on duty at all times. The adjusted odd ratio (OR) was calculated.

All statistical tests were two-sided, and significance was defined as $\mathrm{P}<0.05$. SAS software v9.3 for Windows (SAS Institute, Cary, North Carolina) was used for statistical analyses.

\section{Results}

The facilities had a combined total of 4,963 hospital beds (mean 709, SD 370), 120 of which were ICU beds (mean 17.1, SD 9.2). Of the 120 ICU beds, 70 were mixed general ICU, with the remaining beds classified as "other" types of ICU beds (including 10 medical, 12 surgical, and 28 emergency beds). Two of the ICUs were managed totally by intensivists (closed type ICU) and others were not.

Patients' characteristics and backgrounds are summarized in Table 1. Mean APACHE II score was 23.5 (Max: 31; Min: 19). Overall, approximately half of the patients comprised non-surgical patients including out of hospital or inpatient emergency $(47.5 \%)$ and the remaining were post-surgical patients (52.5\%). Cardiovascular disease was the most frequent cause, accounting for $49.0 \%$, followed by respiratory disease at $17.1 \%$. Patient characteristics between the VAE and No VAE groups are as follows. Proportion of males was high in the VAEs group $(\mathrm{P}=0.043)$, while mean age and average APACHE II score were not statistically significantly different. There was also no significant bias for diagnosis between the two groups.

\section{VAEs incidence}

VAEs incidence and characteristics are displayed in Table 2. We noted 49 total VAEs, which corresponded to $1.1 \%$ of total ICU patients and $5.7 \%$ of patients receiving $\geq 2$ days of MV. The average incidence rate per facility was 7 cases per year. Table 2 also displays the total number of VAC $(n=49)$, IVAC $(n=29)$, and PVAP $(n=16)$ cases. Converting these numbers to $1,000 \mathrm{MV}$ days, the incidences were 6.40 (VAC), 4.19 (IVAC), and 2.29 (PVAP) cases per 1,000 MV days.

With regard to VAE subtypes, separate incidences were determined for VAC only (2.20), IVAC only (1.90), and PVAP (2.29) per 1,000 MV days and were not statistically different $(\mathrm{P}=0.933)$. Of the 49 VAEs, $40.8 \%$ were VAC only, $26.5 \%$ were IVAC only, and $32.7 \%$ were PVAP $(\mathrm{P}=0.322)$ (Table 2). In this study, non-pneumonia (VAC only + IVAC only) accounted for $67.3 \%$ of VAEs. Infectious complications (IVAC only + PVAP) were $59.2 \%$ of VAEs (Table 2). 
Table 1 Characteristics of enrolled patients

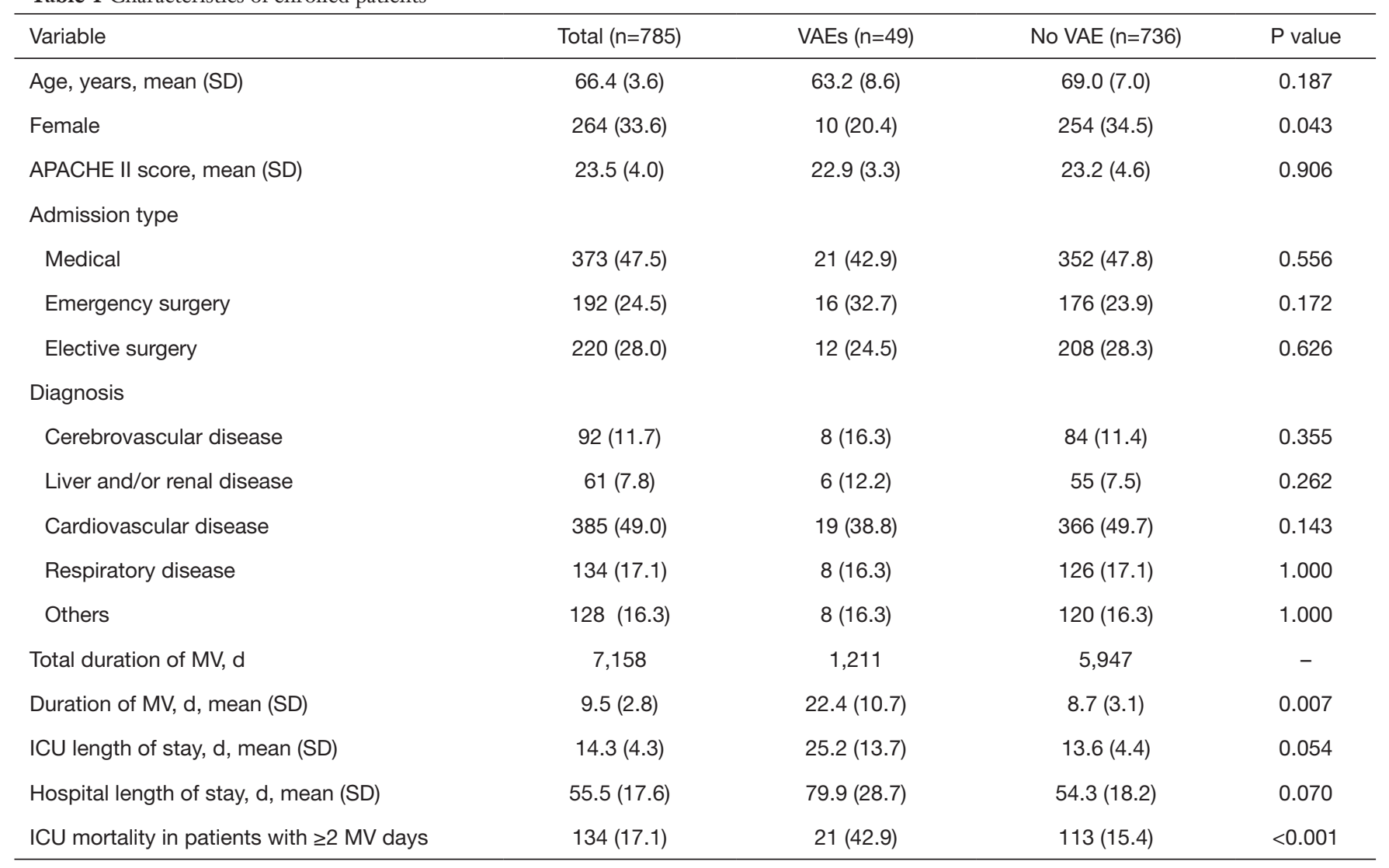

Data are presented as $\mathrm{n}(\%)$, except where noted otherwise. SD, standard deviation; APACHE, Acute Physiology and Chronic Health Evaluation; MV, mechanical ventilation; ICU, intensive care unit.

Table 2 Ventilator-associated events incidence and mortality

\begin{tabular}{|c|c|c|c|c|c|c|}
\hline VAEs & Total number & Mean (SD) & \multicolumn{3}{|c|}{ Incidence rate } & $\begin{array}{c}\text { Mortality, } \\
\text { n (\%) }\end{array}$ \\
\hline IVAC & 29 & $4.1(3.7)$ & $3.3(1.6)$ & $4.19(3.0)$ & - & 12 (41.4) \\
\hline IVAC only & 13 & $1.9(1.6)$ & $1.6(1.2)$ & $1.90(1.6)^{\dagger}$ & $26.5^{\S}$ & $7(53.8)^{\Uparrow}$ \\
\hline
\end{tabular}

Data are presented as $\mathrm{n}$ or mean and $\mathrm{SD}$, except where noted otherwise. ${ }^{\dagger}, \mathrm{P}=0.933 ;{ }^{\S}, \mathrm{P}=0.322 ;{ }^{\uparrow}, \mathrm{P}=0.459 . \mathrm{SD}$, standard deviation; VAE, ventilator-associated event; VAC, ventilator-associated condition; IVAC, infection-related ventilator-associated complication; PVAP, possible and probable ventilator-associated pneumonia; MV, mechanical ventilation.

Figure 2 (left panel) shows VAE incidence across all seven facilities. All facilities had VAE with no statistically significant difference in VAE incidence ( $\mathrm{P}=0.252)$. However, there was variation width in incidence rates. In addition, the pathoetiology of VAEs were facility dependent. For example, pneumonia did not occur in facilities D, F, and $\mathrm{G}$, only pneumonia occurred in facility A, noninfectious complications did not occur in facility $\mathrm{C}$, and noninfectious 

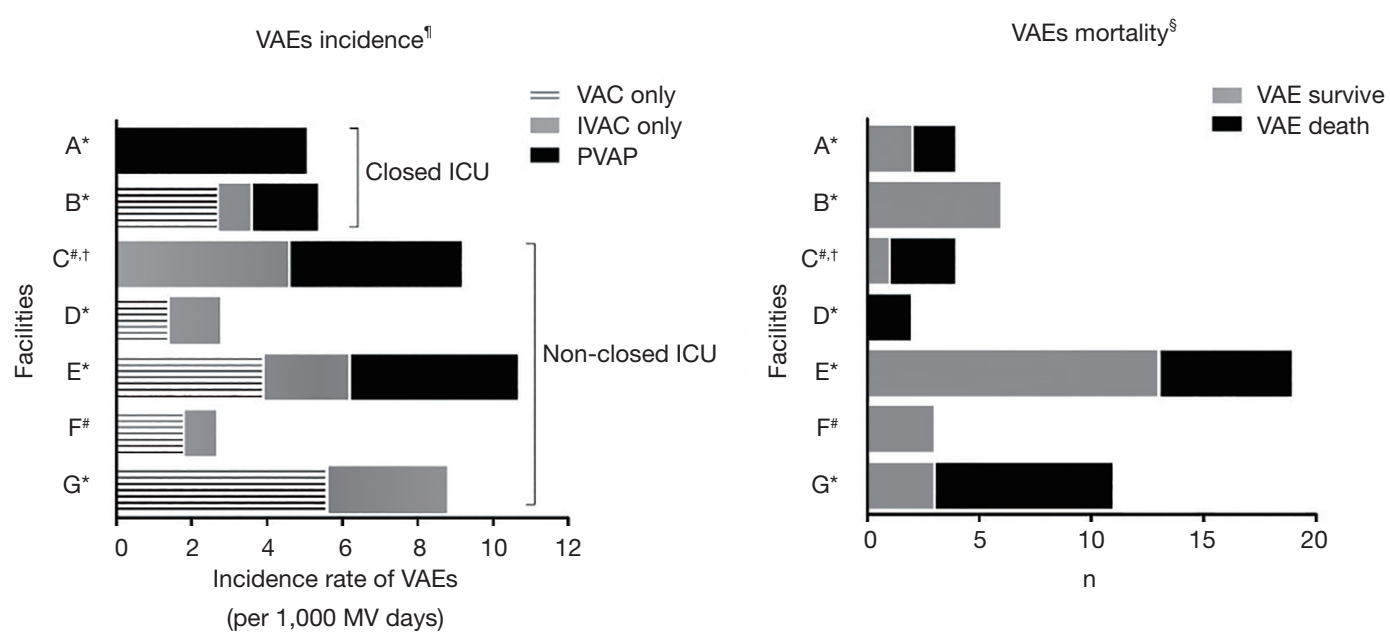

Figure 2 VAEs incidence and mortality in each facility. *, University hospital; ${ }^{*}$, Community hospital; ${ }^{\dagger}$, Hospital with protocol driven mechanical ventilator settings; ", left panel shows that there was no statistically significant difference in the incidence of VAEs between 7 facilities $(\mathrm{P}=0.252)$, however, there was variation width in incidence rate; $\$$, right panel shows that facility B had low VAE mortality, and facility $G$ had high VAE mortality $(\mathrm{P}<0.05$ from multiple testing controlled by the Benjamini-Hochberg procedure). VAE, ventilatorassociated event; VAC, ventilator-associated condition; IVAC, infection-related ventilator-associated complication; PVAP, possible and probable ventilator-associated pneumonia.

Table 3 Generalized linear model (GLM) showing association of VAEs incidence with characteristics of the facilities

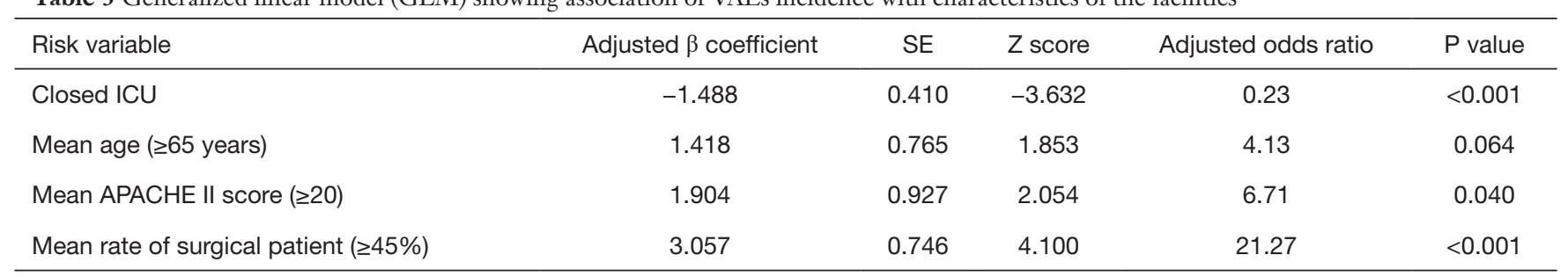

VAE, ventilator-associated event; SE, standard error; APACHE, Acute Physiology and Chronic Health Evaluation; ICU, intensive care unit.

complications mainly occurred in facility G.

The adjusted odds ratios calculated by the generalized linear model (GLM) in order to investigate the relationship between facility features and prevalence of VAEs were as follows: 0.23 for "closed-ICU"; 6.71 for "APACHE II score 20 or more"; and 21.27 for "the ratio of surgical cases to $45 \%$ or more". There was statistical significant difference in each of these factors (Table 3). Incidence of VAEs in ICU was found to increase with a large proportion of surgical patients and more severe patients, while converse decreased in closed ICU.

\section{VAEs outcomes}

Death was observed in 134 (17.1\%) of the ICU 785 patients with $\geq 2 \mathrm{MV}$ days (Table 1). Of these patients, 21 (15.7\%) had VAEs. This reflected a rate of about $3.0 \%$ of patients with $\geq 2 \mathrm{MV}$ days and $0.5 \%$ of total ICU patients. When analyzing the relationship between the number of deaths and development of VAEs, the mortality rate of the VAEs group was $42.9 \%$ (21/49). This was significantly different from mortality rates observed in the No VAE group (15.4\%: 113/736; $\mathrm{P}<0.001$, Table 1). The (non-adjusted) odds ratio for death in the VAE group was 4.13 times that of the No VAE group. Number needed to harm (NNH) was 3.6. There were no differences in ICU mortality among the three subtypes ( $\mathrm{P}=0.459$, Table 2$)$, with all three groups showing a tendency toward high mortality. The mean duration of $M V$ was statistically significantly longer for the VAE group than for the No VAE group (22.4 vs. 8.7, $\mathrm{P}=0.007$ ), and the average length of ICU stay also tended to be longer for the VAE group (25.2 vs. 13.6, $\mathrm{P}=0.054)$. 
Figure 2 (right panel) shows VAE mortality across all seven facilities. Variation width among facilities in VAE mortality was observed. For example, facility B had no VAE death, while facility $\mathrm{G}$ had high VAE mortality rate $(\mathrm{P}<0.05)$.

\section{Discussion}

This preliminary research established the prevalence of VAEs $(5.7 \%$ of patients with $\geq 2 \mathrm{MV}$ days, $6.40 / 1,000 \mathrm{MV}$ days) in Japanese ICU. The presence of VAEs, specifically VAC or IVAC, was related to mortality. We observed the variety of incidence rate, VAE mortality, and its etiology among the centers.

Overall, the low incidence of VAEs in this study is striking. Data from a Canadian study of patients in ICUs with APACHE II scores of 21-23 found an incidence of $11 \%$ for patients with $\geq 2 \mathrm{MV}$ days (17). Zhu et al. reported VAEs in $15 \%$ of patients with $\geq 2 \mathrm{MV}$ days and overall incidence was 11.1/1,000 MV days (19). According to a report by Hayashi et al. conducted in a single center with APACHE II scores of 21-22, VAEs were found in 28\% of the patients with $\geq 2 \mathrm{MV}$ days (11). Compared to these reports with similar APACHE II scores as our study, we found that our VAE incidence was low.

The mortality rate in VAE patients is about $43 \%$, which was not markedly different from that reported in previous investigations, in which the mortality rates were $11 \%$ (11), $25 \%$ (17), and $47 \%$ (19). In addition, there were statistically significant differences in the mortality rates relative to the presence or absence of VAEs, which was similar to previous results $(2,12,15,19,20)$. As important outcomes such as mortality, duration of MV, and length of ICU stay were significantly different between the VAE and No VAE groups, VAEs may have negative consequences for $\mathrm{MV}$ patients. Our study results are based on statistical analysis without controlling for confounding factors, but our cohort has the same background factors such as age, admission type, disease classification, and severity. Thus these results suggest that VAEs may adversely affect $M V$ patients.

In this study, we found that incidence and distribution of VAE subtypes, etiology and mortality were different in each facility. This is important for considering a future VAEs prevention program. There is a potential for variation in the major risks of VAEs among the facilities, depending on the etiological components of clinical VAEs in each ICU. The response to preventive VAE interventions [ex. sedative agents (26), tapered-cuff tracheal tube (27), ventilator bundle (28)] varies depending on the dominant pathoetiological VAE (29). The diverse pathoetiological VAE conditions suggests that directly applying the traditional VAP bundle (30) may not be appropriate when addressing a quality improvement initiative for VAE prevention, most notably for facility D, F, and G. Thus, development of preventive VAE measures should be based on the VAE pathoetiology in each ICU.

Japanese ICUs with a large proportion of surgical patients and more severe cases tended to have increased VAE incidence, with a converse decrease in closed ICUs. This is important when implementing VAEs surveillance as a public reporting system in Japan. Our findings indicated that it is necessary to stratify the data in order to compare incidence rate across multiple centers. Based our results, the following stratification method is suggested for Japan: (I) to categorize facilities based on an average APAHCHE II score that is greater than 20 or less than 20; (II) to categorize by Closed/ Non-closed ICU; (III) and to categorize by surgical/medical ICU. Unlike the United States, functional differentiation of ICU has not progressed in Japan, and stratification by APACHE II score is therefore considered suitable.

Overall, there were no differences in the mortality rates among VAC only, IVAC only, and PVAP as VAE subtype, which is in line with results from Klompas et al. in USA (15). The globally equivalent distribution of the incidence of the three subtypes in this study differs from that in reports of prior studies, where high rates (50-63\%) were observed for VAC only as non-infectious complications $(8,12,15-20)$. The reason for this disparity is unknown; however, the rate of complications of non-pneumonia was $67.3 \%$ of all VAEs in our study, which is in line with rates reported in previous studies $(8,12,15,18-20)$. Our study suggests that targeting pneumonia as an isolated ICU QI is inadequate. The monitoring of "non-infectious complications" and "infectious complications other than pneumonia" may also be important. These findings support shifting the focus away from pneumonia only in initiatives of CDC/NHSN. Our data suggests the importance of surveillance not only pneumonia of but also non-infectious and non-pneumonia factors, broader classification of respiratory deterioration, as a significant associated factor for poor outcome.

It remains uncertain that VAEs could become a potential ICU QI. The prevalence of VAEs is approximately $1.0 \%$ of the total ICU patients in this study, indicating its limited use for QI. It is also important to consider the extent to which QIs contribute to improving outcomes. One study indicates that preventable VAEs comprised only $37 \%$ of the total VAEs (18). If we apply this conclusion to our data, 
preventative efforts may potentially reduce the observed VAEs from 49 to 31 of 4,541 total ICU patients and VAE-associated deaths from 21 to 13 (decrease rate: 1.8 per 1,000 ICU patients and 1.0 per 100 patients with $\geq 2 \mathrm{MV}$ days), an extremely small impact on QI for the overall ICU population (31).

\section{Study limitations}

Because we did not collect detailed patient-specific information, a more elaborate methodology is required to correctly evaluate if VAEs could be a modifiable outcome or just a surrogate of disease severity.

\section{Conclusions}

This study has highlighted some potential of VAEs as a new QI and the challenges facing Japanese ICUs in its use. VAEs may affect ICU mortality rates, however, uncertainty about the extent of the true impact on patients' outcome and a deficit of robust preventive approaches for VAEs would discourage VAE as a QI measure. Although some potential indices of VAEs are suggested to serve as QIs, additional studies to elaborate its practicality or adequacy would further be required.

\section{Acknowledgements}

We would like to sincerely thank all the staff of the Department of Emergency and Critical Care Center of Mie University Hospital, Emergency and Critical Care Center of Tokushima University Hospital, Department of Emergency and Critical Care Center of Hiroshima University Hospital, Intensive Care Unit of Akita University Hospital, Intensive Care Unit of Mitsubishi Kyoto Hospital, Intensive Care Unit of National Hospital Organization Kyoto Medical Center, and Department of Critical Care Center of Fujita Health University Hospital, for their assistance in conducting the study.

Funding: S Nakahashi was supported by a Japan Society for the Promotion of Science (JSPS) KAKENHI Grant Number JP 16K11399. S Ohshimo was supported by JSPS KAKENHI Grant Number JP 16K09541. N Shime was supported by JSPS KAKENHI Grant Number JP 17K11573.

\section{Footnote}

Conflicts of Interest: The authors have no conflicts of interest to declare.

Ethical Statement: Institutional review board approval was obtained for this study (ID: 1468) and we waived the requirement for patient consent because of the retrospective nature of this study.

\section{References}

1. Centers for Disease Control and Prevention (CDC). Surveillance for ventilator-associated events. [updated 2013 Apr 24]; Available online: http://www.cdc.gov/nhsn/ acute-care-hospital/vae/

2. Klompas M, Khan Y, Kleinman K, et al. Multicenter evaluation of a novel surveillance paradigm for complications of mechanical ventilation. PLoS One 2011;6:e18062.

3. Klompas M, Kleinman K, Khan Y, et al. Rapid and reproducible surveillance for ventilator-associated pneumonia. Clin Infect Dis 2012;54:370-7.

4. Klompas M, Magill S, Robicsek A, et al. Objective surveillance definitions for ventilator-associated pneumonia. Crit Care Med 2012;40:3154-61.

5. Klompas M, Berra L. Should Ventilator-Associated Events become a Quality Indicator for ICUs? Respir Care 2016;61:723-36.

6. Magill SS, Fridkin SK. Improving surveillance definitions for ventilator-associated pneumonia in an era of public reporting and performance measurement. Clin Infect Dis 2012;54:378-80.

7. Magill SS, Klompas M, Balk R, et al. Developing a new, national approach to surveillance for ventilatorassociated events: executive summary. Clin Infect Dis 2013;57:1742-6.

8. Magill SS, Li Q, Gross C, et al. Incidence and Characteristics of Ventilator-Associated Events Reported to the National Healthcare Safety Network in 2014. Crit Care Med 2016;44:2154-62.

9. Bouadma L, Sonneville R, Garrouste-Orgeas M, et al. Ventilator-Associated Events: Prevalence, Outcome, and Relationship With Ventilator-Associated Pneumonia. Crit Care Med 2015;43:1798-806.

10. Damas P, Frippiat F, Ancion A, et al. Prevention of ventilator-associated pneumonia and ventilator-associated conditions: a randomized controlled trial with subglottic secretion suctioning. Crit Care Med 2015;43:22-30.

11. Hayashi Y, Morisawa K, Klompas M, et al. Toward improved surveillance: the impact of ventilator- 
associated complications on length of stay and antibiotic use in patients in intensive care units. Clin Infect Dis 2013;56:471-7.

12. Klein Klouwenberg PM, van Mourik MS, Ong DS, et al. Electronic implementation of a novel surveillance paradigm for ventilator-associated events. Feasibility and validation. Am J Respir Crit Care Med 2014;189:947-55.

13. Mekontso Dessap A, Katsahian S, Roche-Campo F, et al. Ventilator-associated pneumonia during weaning from mechanical ventilation: role of fluid management. Chest 2014;146:58-65.

14. Prospero E, Illuminati D, Marigliano A, et al. Learning from Galileo: ventilator-associated pneumonia surveillance. Am J Respir Crit Care Med 2012;186:1308-9.

15. Klompas M, Kleinman K, Murphy MV. Descriptive epidemiology and attributable morbidity of ventilatorassociated events. Infect Control Hosp Epidemiol 2014;35:502-10.

16. Lewis SC, Li L, Murphy MV, et al. Risk factors for ventilator-associated events: a case-control multivariable analysis. Crit Care Med 2014;42:1839-48.

17. Muscedere J, Sinuff T, Heyland DK, et al. The clinical impact and preventability of ventilator-associated conditions in critically ill patients who are mechanically ventilated. Chest 2013;144:1453-60.

18. Boyer AF, Schoenberg N, Babcock H, et al. A prospective evaluation of ventilator-associated conditions and infection-related ventilator-associated conditions. Chest 2015;147:68-81.

19. Zhu S, Cai L, Ma C, et al. The Clinical Impact of Ventilator-Associated Events: A Prospective Multi-Center Surveillance Study. Infect Control Hosp Epidemiol 2015;36:1388-95

20. Sim JK, Oh JY, Min KH, et al. Clinical significance of ventilator-associated event. J Crit Care 2016;35:19-23.

21. Nakahashi S, Yamada T, Ogura T, et al. Association of Patient Care with Ventilator-Associated Conditions in Critically Ill Patients: Risk Factor Analysis. PLoS One

Cite this article as: Nakahashi $\mathrm{S}$, Imai $\mathrm{H}$, Imanaka $\mathrm{H}$, Ohshimo S, Satou T, Shima M, Yanagisawa M, Yamashita C, Ogura T, Yamada T, Shime N; for the VAEs Review Committee in the Japanese Society of Intensive Care Medicine (JSICM). Ventilator-associated events: prevalence and mortality in Japan. J Thorac Dis 2018;10(12):6942-6949. doi: 10.21037/ jtd.2018.12.40 2016;11:e0153060.

22. Benjamini Y, Hochberg Y. Controlling the false discovery rate: a practical and powerful approach to multiple testing. J R Statist Soc B 1995;57:289-300.

23. Committee on ICU Evaluation JSoICM, The Health Labour Science Research group for "DPC" tMoH, Labour, and Welfare. Influence of staffing and administrative policy of ICU on patient outcome. J Jpn Soc Intensive Care Med 2011;18:283-94.

24. Knaus WA, Draper EA, Wagner DP, et al. APACHE II: a severity of disease classification system. Crit Care Med 1985;13:818-29.

25. Treggiari MM, Martin DP, Yanez ND, et al. Effect of intensive care unit organizational model and structure on outcomes in patients with acute lung injury. Am J Respir Crit Care Med 2007;176:685-90.C

26. Klompas M, Li L, Szumita P, et al. Associations Between Different Sedatives and Ventilator-Associated Events, Length of Stay, and Mortality in Patients Who Were Mechanically Ventilated. Chest 2016;149:1373-9.

27. Jaillette E, Girault C, Brunin G, et al. Impact of taperedcuff tracheal tube on microaspiration of gastric contents in intubated critically ill patients: a multicenter clusterrandomized cross-over controlled trial. Intensive Care Med 2017;43:1562-71.

28. Klompas M, Li L, Kleinman K, et al. Associations Between Ventilator Bundle Components and Outcomes. JAMA Intern Med 2016;176:1277-83.

29. Cocoros NM, Klompas M. Ventilator-Associated Events and Their Prevention. Infect Dis Clin North Am 2016;30:887-908.

30. Al-Tawfiq JA, Abed MS. Decreasing ventilator-associated pneumonia in adult intensive care units using the Institute for Healthcare Improvement bundle. Am J Infect Control 2010;38:552-6.

31. Klompas M, Platt R. Ventilator-associated pneumonia-the wrong quality measure for benchmarking. Ann Intern Med 2007;147:803-5. 